\title{
Oxygen-ozone therapy: paradoxical stimulation of ozone
}

\author{
Luigi Valdenassi, ${ }^{1,2}$ Marianno Franzini, ${ }^{1}$ Vincenzo Simonetti, ${ }^{1,3}$ Giovanni Ricevuti ${ }^{4}$ \\ ${ }^{1}$ Oxygen-Ozone Therapy Scientific Society, Gorle (BG); ${ }^{2}$ Department of Internal Medicine \\ and Medical Therapy, University of Pavia; ${ }^{3}$ Kaos non-profit Association, Caselle Torinese (TO); \\ ${ }^{4}$ Department of Internal Medicine, University of Pavia, Italy
}

\begin{abstract}
After several years of scientific research and accurate clinical practice, the oxygen-ozone therapy continues to represent a method full of prospects and new opportunities. On this basis we present an updated review on basic mechanisms through which the ozone acts.

Ozone, a molecule notable for its heterodoxy and atypicalness, has been regarded with suspicious for a long time since it was a possible generator of free radicals and correlated pathologies, but it is important distinguishing between the oxidative damage from free radicals, characterized by irreversible chemical modifications of the molecular target, and the physiological effects from free radicals (redox signaling), characterized on the other hand, by reversible chemical and selective alterations, which are potentially suitable to become an active part in the various metabolic regulation mechanisms, with positive effects on several pathologies.
\end{abstract}

\section{Introduction}

The oxygen-ozone therapy represents a method that after several years of scientific research and accurate clinical practice, continues to prove to be full of prospects and new opportunities. On this basis it is important to draw up an updated review on basic mechanisms through which the ozone acts. The first concept to deal with is the one concerning its influence on the oxidation-reduction processes. ${ }^{1,2}$

Being the ozone an oxidizing molecule, it has been regarded with suspicious for a long time since it was a possible generator of free rad-

Correspondence: Luigi Valdenassi, Department of Internal Medicine and Medical Therapy, University of Pavia, via Aselli 543/45, 27100 Pavia, Italy. E-mail: luigi.valdenassi@unipv.it

Key words: Oxygen-ozone therapy; free radicals; redox signaling.

Received for publication: 12 January 2016.

Accepted for publication: 24 February 2016.

(C) Copyright L. Valdenassi et al., 2016

Licensee PAGEPress, Italy

Ozone Therapy 2016; 1:5837

doi:10.4081/ozone.2016.5837

This article is distributed under the terms of the Creative Commons Attribution Noncommercial License (by-nc 4.0) which permits any noncommercial use, distribution, and reproduction in any medium, provided the original author(s) and source are credited. icals and correlated pathologies. Actually we should more correctly refer to it as paradoxical stimulation of ozone because even if it is an oxidizing molecule, it increases the antioxidant properties of those structures affected by its activity. 2,3

And exactly on a controlled and moderated oxidative stress produced by the reactions of ozone with the different biological components of the organism is based the efficacy of the oxygen-ozone therapy. 3,4

\section{Biologically important free radicals}

It is necessary to point out the fact that until the second half of the $20^{\text {th }}$ century, the research on the forms of free radicals made this name a synonym of harmful molecular events. Only in the first years of the new century, the new knowledge substantially changed such concept, conferring on free radicals a functional potential permeating almost all biological phenomena. ${ }^{5}$

Therefore we have the evidence of free radicals with biological significance (redox signaling), playing an important role in the transmission of signals between cells and tissues for the regulation of organic functions. ${ }^{6}$ Although it was previously pointed out that biological oxidation-reduction processes involving the exchanges between oxidizing and reducing agents occur through the activity of free radical intermediates: the semiquinones.

This leads us to distinguish between the oxidative damage from free radicals, characterized by irreversible chemical modifications of the molecular target that is indiscriminately hit compared to the type of molecule and chemical group that are modified, and the physiological effects from free radicals, characterized on the other hand, by reversible chemical and selective alterations, which are potentially suitable to become an active part in the various metabolic regulation mechanisms. ${ }^{1,5}$

Ozone is a molecule heavier and 10 times more soluble in water than oxygen, and this is important to understand the rapidity with which its reactions occur. Moreover, it is not a radical molecule, however it can be regarded as one of the main oxidants. When coming into contact with plasma water, while the oxygen has a very low solubilization and only saturates haemoglobin, which is partially oxygenated, the ozone instantly reacts with different reducing molecules such as unsaturated fatty acids (PUFAs), different antioxidants and other substances, generating extremely reactive oxygen atoms. ${ }^{5,7,8}$

The ozone, by reacting with the double bonds of unsaturated fatty acids, generates two molecules of aldehyde, called LOPs or lipid oxidation products and hydrogen peroxide. Even if this compound is not a free radical molecule represents a reactive oxygen species (ROS) like the nitroxide (NO) and exactly $\mathrm{H}_{2} \mathrm{O}_{2}$ and $\mathrm{NO}$ are the fundamental reactive species for the biological activation reactions. ${ }^{9}$

The aldehyde and free radical molecules toxicity is widely diluted by 
their distribution in biological liquids, by the enzymatic inactivation performed by the glutathione (GSH)-transferase and by the aldehyde dehydrogenase constituting an important protection system to buffer the oxidative excess by reducing the lipid oxidation products to infinitesimal, non-toxic and much more useful levels, because of their activity as second messenger informing the different organs about an incipient oxidation and thus starting the defensive activation., ${ }^{4,6}$

This determines an immediate increase in the synthesis of antioxidant enzymes: catalase, superoxide dismutase, GSH-peroxidase, as well as the activation of nuclear related factor 2 (Nrf2), a factor present in the protection against neurodegenerative pathologies. Furthermore it is noticed a suppressive tendency towards the $\mathrm{kB}$ nuclear transcription factor (NFkB) which is at the root of the activities carried out by COX2, PGE2 and by other different pro-inflammatory cytokines. ${ }^{10,11}$

\section{Bio-regulating activity}

The Nrf2 determines the induction of heme oxygenase 1, an enzyme responding to stress and converting heme into bilirubin (a real lipophilic antioxidant aimed at interrupting the lipid oxidation), of carbon monoxide (adjuvant of nitric oxide in vasodilation) and free iron. Such enzyme has important antinflammatory and anti-apoptotic effects revealed in some types of ischaemia reperfusion in rats and pigs where its impact on post-ischemic inflammation and its heart protection activity has been assessed. ${ }^{12,13}$

This mechanism represents the basis of the comprehension of the paradoxical phenomenon for which an oxidizing molecule triggers off, on the contrary, a strongly antioxidant reaction. ${ }^{4}$

Obviously, in order to obtain a therapeutic effect rather than a harmful one from oxidative stress induced by ozone, it shall be assessed precisely through very well defined concentrations and quantities. ${ }^{10}$

Only within a selected ambit, called therapeutic window, it can have a real clinical efficacy by inducing a stability in the redox balance and conferring on cell a greater resistance to oxidative insults. ${ }^{14}$ This must take place according to specific selections concerning the type of treated pathology, its stage and the characteristics of each treated subject. ${ }^{4,10}$

Since the mixture clinically used is made up of oxygen and ozone, these two gases come into contact with biological liquids, and while the first one finds a certain balance also in the intracellular diffusion, the second one instantly reacts with PUFAs, different hydrophilic and lipophilic compounds, that, through the transfer of electrons, almost completely neutralize it. Hence the need to use doses able to enable the ozone to generate ROS and LOPS depending on the substrates ability to reduce them. ${ }^{15}$

The ROS activity as well as the hydrogen peroxide (produced after the rapid and controlled stimulation induced by ozone), show itself through their clear increase in plasma, from which they can enter by crossing the cell membranes, into the haematic cell cytoplasm. All this occurs under the control of several intracellular antioxidants such as GSH, catalase and GSH-peroxidase preventing the occurrence of oxidative damage to cellular components. ${ }^{6,9,12}$

Therefore it is in plasma and in interstitial fluids that ozone depletes after having come into contact with a wide variety of biomolecules.

\section{Influence of ozone on blood corpuscular parts}

In erythrocytes GSH is oxidized through the transformation of $\mathrm{H}_{2} \mathrm{O}_{2}$ into $\mathrm{H}_{2} \mathrm{O}$ and, therefore by creating an imbalance of the relation between GSH and glutathione disulfide (GSSG) which is however restored very quickly through the GSH-reductase reducing again the GSSG to GSH and thanks to the prompt usage of nicotinamide adenine dinucleotide phosphate coming from the G6P oxidation. Such reactions determine an increase in glycolysis and in the bioavailability of ATP included in the wider framework of bioenergetic activities performed by ozone. ${ }^{13,15}$

The stimulation does not just act on erythrocytes but, by coming into contact with leucocytes determines an increase in the phagocytic activity of neutrophils, while in lymphocytes, the entry of hydrogen peroxide activates the enzymatic groups causing the phosphorylation of IkB proteins that dissociate from NFkB allowing other biological agents to create a bond with specific DNA sites, and this is very useful to regulate the gene expression. NFkB represents an important transcription factor modulated by hydrogen peroxide, which in turn is responsible for the production of different cytokines (interleukin-1, $2,-6,-8$, interferon $\beta$ and tumor necrosis factor $\alpha$ ) but also by GSH and other reducing compounds. This emphasizes how a transitory increase in ROS can turn out to be useful in the expression of immune-modulating activities. ${ }^{16,17}$

We saw how ozone interacts with erythrocytes and leucocytes; in platelets, ROS determine the release of ATP and of other growth factors: ${ }^{18}$ i) platelet derived growth factor; ii) transforming growth factor $\beta$; iii) basic fibroblast growth factor; iv) hepatocyte growth factor; v) epidermal growth factor; vi) vascular endothelial growth factor.

Their clinical application becomes more and more important, since they are suitable to stimulate cell proliferation and differentiation. Basically they are molecules used for the communication among the different cells of an organism. ${ }^{6,13}$

Their main functions are the external control of the cell cycle, the regulation of their entry into mitosis, cell survival, cell migration and differentiation. It is important to remind that proliferation and differentiation are simultaneously activated otherwise there would be the onset of a tumour.

This clarifies the benefit of ozone therapy in the treatment of trophic lesions and of ulcers both in diabetic subjects and in case of arteriophaties and venous insufficiencies where it allows a vascularization improvement, induces the tissue granulation and a consequent quick cicatrization of lesions. ${ }^{15}$

To summarize we can say that the action of ozone mainly takes place in two stages: the first one is very rapid and is due to the ROS, the second one involves the LOPs, diffusible compounds that are induced by the contact between ozone and blood and, in the return stage, pass through the endothelium and then dilute in interstitial fluid up to the different cells of the organism, carrying out a messenger activity while informing cells about the onset of repeated, even if controlled, oxidative stress. ${ }^{4}$

\section{Ozone and nitroxide}

What is here described points out the abundance of activities and biological compounds involved, that bond to different receptors following the paradoxical stimulation induced by the ozone used to normalize or reactivate the basic biological phenomena. The endothelium, after its interaction with LOPs through the activation of NO-synthetase, induces the release of $\mathrm{NO}$ and nitrosothiols. ${ }^{19,20}$

The latter consists in organic compounds, both existing in the organism (endogenous) and external (exogenous), containing a nitrous group (NO) attached to the sulphur atom of a thiol (a substance which in turn contains an SH group, for example cysteine, a type of amino acids contained in glutathione). Such substances are very important because they transfer to some proteins the nitro group 
NO, useful for regulating their function, as well as the maturation processes and the interactions with other proteins. Furthermore they have an antioxidant activity, which regulates the inflammatory processes.

According to nitroxide, a chemical species with free radical origin considered for many years to be a highly toxic gas, and that only recently has been identified as a powerful chemical mediator able to control several biological functions, we recall Luis Ignarro who was awarded the Nobel prize for his studies on this signaling molecule. ${ }^{19}$

An ubiquitous molecule acting as important intra- and intercellular messenger regulating multiple functions among which the vascular endothelium one. It has been noticed, on human endothelial cells culture briefly exposed to ozonated plasma, a greater production of NO and this suggests a real induction of NO-synthetase, an enzyme transforming the L-arginine into L-citrulline and consequent NO liberation.

This, by acting on the receptors of smooth muscle cells subsequently activates the guanylate cyclase, an enzyme intervening in cyclization of guanosine triphosphate generating the cyclic guanosine monophosphate (cGMP).

It is proved that the NO: reduces the platelet aggregation, the leucocyte adhesion to vasal walls and the relaxation of smooth muscles. Consequently, significant anti-aggregant, anti-inflammatory and antihypertensive effects are generated, making this molecule extremely important to such an extent that its reduced bioavailability is regarded to be responsible for the onset and worsening of several pathologies such as the arterial hypertension and atherosclerosis.

NO is the main second messenger generated by it, cGMP in fact shows a fundamental role in the physiological control of immune response and in the central and peripheral neurohumoral transmission.

cGMP has a protein kinase aimed at phosphorylating some target proteins with their relevant functions: i) myocardial proteins involved in calcium homeostasis; ii) proteins involved in the onset of programmed apoptosis; iii) control capacity on smooth muscle contractility; iv) control on ionic influx into retinal cells; v) memory learning and consolidation phenomena; vi) protection of nerve cells from neurodegeneration; vii) modulation of different immune responses.

cGMP is then hydrolyzed and then inactivated by phosphodiesterase among which the type 5 (PDE5) is the target of sildenafil, the principle used in erectile dysfunctions. This is a PDE5 inhibitor, therefore a preservation of high cGMP concentrations determines a local vasodilation with a greater hematic flow useful to maintain erection.

\section{Conclusions}

This captivating context of biological activities with the relevant relapses in the clinical setting, make more and more interesting the research pathway concerning ozone, a molecule notable for its heterodoxy and atypicalness, the foundations on the basis of its influence on the preservation of an adequate stability in the redox balance from which important and effective effects ensue, on pathologies which are very different from one another that may find in this molecule an unexpected bioregulation element. ${ }^{4,10}$

\section{References}

1. Duan X, Buckpitt AR, Plopper CG. Variation in antioxidant enzyme activities in anatomic subcompartments within rat and rhesus monkey lung. Toxicol Appl Pharmacol 1993;123:73-82.

2. Muñoz A. Design and analysis of studies of the health effects of ozone. Environ Health Perspect Suppl 1993;101:231-s5.

3. Plopper CG, Duan X, Buckpitt AR, Pinkerton KE. Dose-dependent tolerance to ozone. IV. Site-specific elevation in antioxidant enzymes in the lung of rats exposed for 90 days or 20 months. Toxicol Appl Pharmacol 1994;127:124-31.

4. Bocci V, Borrelli E, Travagli V, Zanardi I. The ozone paradox:ozone is a strong oxidant as well as a medical drug. Med Res $\operatorname{Rev} 2009 ; 29$ : 646-82.

5. Bocci V. Ozone therapy today. Proc. 12th World Congress of the International Ozone Association. Ozone in Medicine. Lille: ToursInstaprint; 1995. pp 13-27.

6. Stone JR, Yang S. Hydrogen peroxide: a signaling messenger. Antioxid Redox Signal 2006;8:243-70.

7. Rilling SH. The basic clinic applications of ozone therapy. OzoNachrichten 1985;1/2:7-17.

8. Viebahn R. The use of ozone in Medicine. 2nd. Rev. Berlin: Haugh Publ. Ed.: 1994. pp 7, 22, 100.

9. Antunes F, Cadenas E. Estimation of H2O2 gradients across biomembranes. FEBS Lett 2000;475:121.

10. Bocci V, Zanardi I, Travagli V. Ozone: a new medical drug in vascular diseases. Am J cardiovasc Drugs. 2011.

11. Mendiratta S, Qu ZC, May JM. Erytrocyte ascorbate recycling. Antioxidant Free Radic Biol Med 1998;24:789-97.

12. Stone JR, Collins T. The role of hydrogen peroxide in endothelial proliferative responses. Endothelium 2002;9:231-8.

13. Razumovskii SD, Konstantinova ML, Grinevich TV, et al. Mechanism and kinetics of the reaction of ozone with sodium chloride in acqueous solutions. Kinet Catal 2010;51:492-6.

14. Valdenassi L, Richelmi P, Bonetti M, et al. Studio sperimentale sulla stabilità dell'ozono nelle usuali condizioni di utilizzo. Atti: World Congress on Oxygen Ozone Therapy -Tertium Millenium, March 11-13, 1999, Verona, Italy.

15. Franzini M, Bignamini A, Micheletti P, et al. Subcutaneous oxygenozone therapy in indurative hypodermatitis and in localized lipodystrophies. A clinical study of efficacy and tolerability. Acta Toxicol Ther 1993;14:273-88.

16. Valacchi G, Bocci V. Studies on the biological effect of ozone: 11. Release of factors from human endothelial cells. Mediat Inflamm 2000;9:271-6.

17. Molinari F, Simonetti V, Franzini M, et al. Ozone autohemotherapy induces long-term cerebral metabolic changes in multiple sclerosis patients. Int J Immunopathol Pharmacol 2014;27:379-89.

18. Valacchi G, Bocci V. Studies on the biological effects of ozone: 10. Release of factors from ozonated human platelets. Mediat Inflamm 1999;8:205-9.

19. Pryor WA, Houk KN, Foote CS, et al. Free radicals - Biology and medicine: it's gas, man!. Am J Physiol 2006;291:R491-511.

20. Nakao A, Sugimoto R, Billiar TR, McCurry KR. Therapeutic antioxidant medical gas. J Clin Biochem Nutr 2009;44:1-13. 